equivocal evidence indicates the influence of marketing on sugary food and drink sales and consumption. However, little research has explored this relationship for SSBs, among adult samples, or using diverse marketing exposures. There are no cross-country comparisons of this relationship.

Aim We aim to answer:

i. Does SSB consumption vary according to self-reported exposure to sugary drink marketing?

ii. Does the relationship between SSB consumption and selfreported exposure to sugary drink marketing vary by country?

Methods We analysed data from the 2017 International Food Policy Study (IFPS), a cross-sectional, online survey that sampled adults aged 18 to 64 years in Australia $(n=3,767)$, UK $(n=4,047)$, Mexico $(n=4,057)$, USA $(n=4,868)$, and Canada ( $n=2,139$; aged 31 to 64 years). Surveys covered eating patterns, food environments, purchasing, knowledge of and attitudes to a range of policy measures. This included a sevenday Beverage Frequency Questionnaire. Dependent (SSB consumption) and independent variables (amount of self-reported sugary drinks marketing exposure) were computed, and potential confounders identified. A multinomial logistic regression model examined relative odds of non-, low- or high- weekly SSB consumption by marketing exposure, country, sex, age, and work status. The same model was reconstructed with interaction between marketing exposure and country, and a log likelihood test was used to compare the models.

Results SSB consumption varied according to self-reported exposure to sugary drinks marketing. 57\% of respondents consumed SSBs, and $72 \%$ self-reported exposure to sugary drinks marketing. Compared to those reporting no sugary drinks marketing exposure, those reporting greater exposure were more likely to have low- than no-SSB consumption and more likely to have high- than no-SSB consumption (ps<0.05). Respondents in Mexico (relative to Canada) and men (vs women) also reported higher sugary drinks consumption, whereas those from the UK, USA (relative to Canada), and older respondents reported lower consumption (ps<0.05). The relationship between self-reported sugary drinks marketing exposure and SSB consumption varied by country $(\mathrm{p}=0.0286)$. Discussion SSB consumption was positively associated selfreported exposure to sugary drinks marketing in all countries, but this relationship varied by country. These analyses are cross-sectional, but the IFPS panel will be re-contacted annually enabling future longitudinal analyses. Improving understanding of the influence of marketing on SSB consumption could help policymakers to augment current strategies to reduce SSB consumption.

\section{RF37 PREDICTORS OF LONELINESS AMONG OLDER MEN AND WOMEN IN ENGLAND}

H Yang, PA Bath*. Information School, The University of Sheffield, Sheffield, UK

\subsection{6/jech-2018-SSMabstracts.125}

Background Loneliness has been considered as a serious social and public health problem among older people. Understanding factors that affect loneliness among older adults is particularly important. However, Loneliness is a complex and multidimensional construct, which is associated with a wide variety of factors. A consideration of multiple dimensions is essential to gain a complete picture of an individual's state of loneliness.

Objective The objective of this study is to investigate the prospective impact of a wide range of factors on loneliness with a focus on: (a) which factors are closely related to the feelings of loneliness, and whether they perform consistently as people age, and (b) whether men and women differ in loneliness experiences.

Methods Data used in the study were from a population-based sample of 3838 core members who participated in all the waves of the English Longitudinal Study of Aging (ELSA) (2002-2015). Binary logistic regression analyses were conducted to understand the relationship of loneliness and associated factors on two sets of experiments: (a) four different wave points with a 4-year measure span were selected to examine the effect of different groups of factors on loneliness prediction and potential prediction changes when ageing. (b) The predictive models were built on separate gender datasets to investigate whether the risk factors for men and women were different. Statistical software SPSS (version 23) was used in the analyses.

Results Variables in the models included: gender, age, marital status, closeness to spouse, contact with children and friends, money shortage, health problem, and depression. The results on four wave points showed that widowhood, emotionally distant to spouse, and depression displayed consistently significant association with loneliness. But contact with friends, money shortage and health problem only showed significance on one or two waves. It suggested there might be changes in risk factors for loneliness as people age. The results on separate gender data confirmed that being widowhood or not close to partner led to a higher risk of being loneliness for women, while health problem and infrequent contact with friends increased the feelings of loneliness in men.

Conclusions This study identified important risk factors influencing loneliness experience. The findings from the study showed some evidence that the impact of risk factors might change when ageing or in different gender. Knowledge about such difference will be helpful in the development of targeted interventions to fight against loneliness in later life.

\section{RF38 'I'VE ALWAYS DONE WHAT I WAS TOLD BY THE MEDICAL PEOPLE' - A MIXED METHODS STUDY OF OLDER PEOPLE'S REASONS FOR ATTENDANCE AT A NEW FALL PREVENTION CLINIC}

${ }^{1} \mathrm{~S}$ McHugh, ${ }^{1} \mathrm{E}$ Racine*, ${ }^{2} \mathrm{~F}$ Cronin, ${ }^{2} \mathrm{~L} O$ O'Sullivan, ${ }^{3} \mathrm{E}$ Moriarty, ${ }^{2} \mathrm{~S}$ Turvey, ${ }^{4} \mathrm{P}$ Barry, ${ }^{5} \mathrm{~K}$ O'Connor, ${ }^{6} \mathrm{~S}$ Timmons, ${ }^{1} \mathrm{PM}$ Kearney. ${ }^{1}$ School of Public Health, University College Cork, Cork, Ireland; ' ${ }^{2}$ St Finbarr's Hospital, Cork, Ireland; ${ }^{3}$ Health Service Executive, Cork, Ireland; ${ }^{4}$ Cork University Hospital, Cork, Ireland; ${ }^{5}$ Mercy University Hospital, Cork, Ireland; ${ }^{6}$ Centre for Gerontology and Rehabilitation, University College Cork, Cork, Ireland

\subsection{6/jech-2018-SSMabstracts. 126}

Background Falls are one of the most serious and common threats facing older adults. Multifactorial falls risk assessment can reduce the rate of falls among community dwelling older adults and is recommended as a first step in the early detection and management of risk factors. It is used as a gateway for older people to access evidence-based interventions targeting their individual risk factors. However, effective interventions will only reduce the rate and risk of falls if there is a high level of uptake in the population and suboptimal uptake 
is a common challenge. The aim of this study is to understand the reasons for the patterns of uptake of multifactorial fall risk assessment clinics in the community.

Methods A convergent mixed methods study design was used. Data on attendance/non-attendance during the first 12 months at each clinic $(n=4)$ were extracted from a centralised administrative database. Semi-structured interviews (n-16) were conducted with a purposive sample of people who attended a clinic (criteria: male/female, age: 55-69, 70-79, 80+, and clinic), selected from those who had opted in during a preceding service-user experience survey. Administrative data were analysed descriptively using STATA (v13) software. Thematic analysis was performed and themes were mapped onto the Theoretical Domains Framework (TDF). Data were merged during analysis and interpretation using qualitative themes to expand on the patterns emerging in the quantitative data.

Results Of the 588 older adults invited, 484 attended the assessment which resulted in an uptake rate of $82.3 \%$ (range: 77.5-86.1). Reasons for attending were summarised into themes which mapped onto 10 theoretical domains. Key themes identified were always attending medical appointments and adhering to medical advice (social/professional role and identity), having a positive attitude towards health services (optimism), being linked in with health professionals and community services (environmental context and resources) and having strong social support from family and friends (social influences). Other themes included understanding what the clinic was about (knowledge), having a fear of falling (emotion), supposing the benefits of attending (beliefs about consequences) and wanting to prevent further falls (goals).

Conclusion The results suggest older people attend fall prevention services if they have existing (positive) links to health services and are well supported in their homes and communities. To improve the spread of fall prevention programmes, there is a need to reach older adults who are less well supported.

\section{Poster presentations}

\section{P1 URBAN LANDSCAPES, CITY DIVERSITY AND QUALITY OF LIFE: AN OBJECTIVE CROSS-SECTIONAL STUDY OF 66 EUROPEAN CITIES}

JR Olsen, N Nicholls, R Mitchell*. MRC/CSO Social and Public Health Sciences Unit, University of Glasgow, Glasgow, UK

\subsection{6/jech-2018-SSMabstracts. 127}

Background With more than half the world's population residing in urban areas and this proportion rising, it is important to understand how urban environment might create happier and healthier populations. Most studies consider the influence of single environmental characteristics, often comparing small numbers of cities. In this novel international study, we applied methods from ecology to assess 66 entire European cities as 'urban landscapes'. We explored relationships between objective measures of the cities' land cover and structure, and residents' reported satisfaction with their life and city. We then assessed whether city landscape characteristics were related to within-city socio-economic inequality in life satisfaction.

Methods Respondents to the European Urban Audit (EUA) report their satisfaction with: life; the city they live in; and the place they live, as well as socio-demographic characteristics. The European Urban Atlas provides satellitederived land use data for these cities. The Atlas has a $10 \mathrm{~m}^{2}$ resolution and categorises land into 26 classes including, for example, industrial sites, transport infrastructure, and different kinds of natural space. The proportion of each city's land area falling within each class, together with metrics quantifying the distribution, balance and spatial arrangement of different land uses, were derived and joined to the EUA ( $n=\sim 63000$ individuals, within 66 cities). Mixed binary logistic analysis sought associations between life satisfaction measures and the land use measures and metrics. Then, associations between a city-level slope index of socio-economic inequality in satisfaction and the landscape metrics were explored using ANCOVA.

Results Specific land use measures were associated with all of the satisfaction measures: the residential proportion of a city (OR: 0.980-0.990, $\mathrm{p}<0.05$ ); continuous urban fabric (OR: $0.995-0.998, \mathrm{p}<0.0 .03$ ); isolated structures (OR: 1.0451.097, $\mathrm{p}<0.05)$; other roads and associated land (OR: 0.967-0.989, p<0.04); pastures (OR: 1:003-1:007, p<0.02) and herbaceous vegetation (OR: $0.990-0.998, p<0.03$ ). No associations were found with the landscape metrics. More diverse landscapes $(\beta:-0.125--0.305, \mathrm{p}<0.001)$, and those with more even distributions of land uses $(\beta$ : $0.397 \quad$ $-1.054, \mathrm{p}<0.001$ ), were both associated with lower inequality in life satisfaction.

Conclusion The content and form of a whole city may influence how residents feel about their lives. Cities which have a diverse range of land use, and which have a more equal balance in land seem also to enjoy lower levels of socio-economic inequality in life satisfaction. These findings may aid urban planners to develop and build cities that can contribute to improving population life satisfaction and narrowing inequalities.

\section{P2 WHAT FACTORS PREDICT PARTICIPATION IN A MASS COMMUNITY PHYSICAL ACTIVITY PROGRAMME? THE CASE OF THE FIVE SHEFFIELD 'PARKRUNS'}

${ }^{1}$ EC Goyder*, ${ }^{1} \mathrm{C}$ Edmonds, ${ }^{1} \mathrm{~A}$ Sabey, ${ }^{1} \mathrm{D}$ Lawrence, ${ }^{2} \mathrm{~A}$ Bullas, ${ }^{1} \mathrm{M}$ Taylor, ${ }^{1} \mathrm{~S}$ Potter. ${ }^{1}$ SCHARR, University of Sheffield, Sheffield, UK; ${ }^{2}$ CSER, Sheffield Hallam University, Sheffield, UK

\subsection{6/jech-2018-SSMabstracts. 128}

Background Community-based 5 kilometre running/walking events known as 'parkruns', organised by volunteers using web based infrastructure maintained by a national charity, are a rapidly growing international phenomenon. Every Saturday morning over 100000 people take part in a 'parkrun' in the UK, with 1200 participating each week in the city of Sheffield. Despite increasing support for events to be set up in public parks in more deprived neighbourhoods, individuals living in less affluent areas are less likely to participate. Better understanding is needed of what makes events accessible and attractive to those from poorer neighbourhoods if there is to be a positive impact on inequalities in physical activity and associated wellbeing. This study explored differences in participation and factors that affect participation across the five Sheffield parkrun events.

Methods We collected qualitative and quantitative information from routine data sources, from the event websites and from social media and pairs of researchers conducted structured observations at each of five events at different locations across 